Revista da Sociedade Brasileira de Medicina Tropical

Journal of the Brazilian Society of Tropical Medicine

Vol.:52:e20190182: 2019

doi: 10.1590/0037-8682-0182-2019

\title{
Editorial
}

\section{Fighting Rheumatic Heart Disease: What are the next moves?}

\author{
Bruno Ramos Nascimento ${ }^{[1],[2]}$ and Andrea Zawacki Beaton ${ }^{[3],[4]}$ \\ [1]. Serviço de Cardiologia e Cirurgia Cardiovascular e Centro de Telessaúde, Hospital das Clínicas, \\ Universidade Federal de Minas Gerais, Belo Horizonte, MG, Brasil. \\ [2]. Departamento de Clínica Médica, Faculdade de Medicina, Universidade Federal de Minas Gerais, Belo Horizonte, MG, Brasil. \\ [3]. The Heart Institute, Cincinnati Children's Hospital Medical Center, Cincinnati, OH, USA. \\ [4]. Division of Pediatrics, The Univeristy of Cincinnati School of Medicine, Cincinnati, OH, USA.
}

Rheumatic heart disease (RHD) is the late sequelae of inadequately treated group A streptococcal pharyngeal infections (GAS), resulting in episodes of acute rheumatic fever (ARF), a multisystem and multifactorial immune response that often culminates in valvular damage and, ultimately, heart failure ${ }^{1}$. In this review, Leal and cols provide a broad overview of the disease, from the global burden and epidemiological aspects to preventive measures and treatment, highlighting the need for sensitization at all levels for the continuing development of broad public policies to confront RHD.

This review article is timely; RHD is reemerging as a global health priority championed by frontline healthcare providers, researchers, and persons living with RHD. In May 2018, the member states of the World Health Organization (WHO) unanimously adopted a Global Resolution on RHD, whose passage represents years of advocacy and campaigning from RHD champions around the globe, but this is only the beginning. The urgent challenge now is to translate this resolution into action by the member states, WHO, and civil societies (Figure 1).

The challenges of RHD management start with its complex pathophysiology, depicted by the authors. Some mechanisms of RHD are well known, such as the triggering of an exaggerated immune response due to antigenic similarity between agent structures (e.g. surface protein $\mathrm{M}$ and the GlcNAc epitope) and molecules in host tissues. In addition, contact between GAS and the subendothelial collagen matrix, with consequent

Corresponding author: Bruno Ramos Nascimento.

e-mail: ramosnas@gmail.com

Orcid: 0000-0002-5586-774X

Received 6 May 2019

Accepted 9 May 2019 binding between $\mathrm{M}$ proteins and the $\mathrm{CB} 3$ region of type IV collagen, results in the generation of a "neo-antigen". However, despite these mechanisms being accepted as the basic triggers of RHD, individual susceptibility is not yet understood. As the authors state, genetic determinants are highly likely, as suggested by studies involving family members and twins, possibly associated with polymorphisms in genes coding for inflammatory cytokines. However, results of genome-wide studies are still preliminary, and some proposed associations between immunoglobulin heavy chain alleles and human leukocyte antigen regions with RHD need further clarification ${ }^{2,3}$. Most importantly, the prognostic role of genetic polymorphisms, which could potentially impact the management of subclinical and clinical disease, is yet to be clarified.

Furthermore, as discussed by the authors, the probability of developing ARF and RHD after a GAS infection is also influenced by bacterial virulence, resulting from a complex interaction between climatic factors, behavioral patterns, and geographic issues - besides the aforementioned genetic predisposition. As examples of this complexity, the prevalence of GAS among children with pharyngitis may vary among countries with similar development backgrounds ${ }^{4,5}$, and previous studies suggested that other predictors of RHD, such as persistently elevated anti-streptolysin $\mathrm{O}$ titers, do not necessarily parallel with socioeconomic status ${ }^{4}$. Interestingly, besides the known social determinants, some GAS emm clusters may have tropism for defined geographical areas ${ }^{4,5}$.

Leal and cols also highlight the growth of RHD research in recent years, boosted by echocardiographic screening studies and frequently accompanied by interventions focused on education, population awareness, and primary prevention. While the real utility of active RHD surveillance through simplified echocardiography is yet to be determined, such worldwide 


\begin{tabular}{|c|c|}
\hline Member States & $\begin{array}{l}\text { - Improve access to primary healthcare. } \\
\text { - } \quad \text { Strengthen data collection and knowledge of RHD prevalence } \\
\text { - } \quad \text { Ensure affordable and reliable access to technologies and } \\
\text { medicine. } \\
\text { - } \quad \text { Ttrengthen national and international cooperation. } \\
\text { Tackle the root determinants of ARF \& RHD. }\end{array}$ \\
\hline Stakeholders & $\begin{array}{l}\text { Put people living with RHD at the center of the prevention and } \\
\text { control agenda. } \\
\text { - Raise the profile of PLWRHD and other NCDs of children and } \\
\text { adolescents on the global health agenda. } \\
\text { Support and facilitate timely, affordable and reliable access to } \\
\text { existing and new cost-effective medicines and technologies for } \\
\text { the prevention and control of RHD. }\end{array}$ \\
\hline WHO & $\begin{array}{l}\text { - } \quad \text { Lead and coordinate global efforts to prevent and control RHD. } \\
\text { - } \quad \text { upport Member States to measure their RHD burdens and } \\
\text { - } \quad \text { Foster international partnerships. } \\
\text { - } \quad \text { Monitor efforts for the prevention and control of RHD. } \\
\text { - } \quad \text { Report on implementation of this resolution to the } 74 \text { th World } \\
\text { Health Assembly, which will be held in } 2021 \text {. }\end{array}$ \\
\hline
\end{tabular}

FIGURE 1: Summary of the WHO Global Resolution on RHD. ARF: acute rheumatic fever; NCD: non-communicable diseases; PLWRHD: people living with rheumatic heart disease; RHD: rheumatic heart disease; WHO: World Health Organization.

programs have proven useful for advocacy and awareness about the epidemiological significance of the disease, thereby contributing to bring this topic to policymakers. More than screening itself, many of these programs have implemented educational curriculums to children, school and healthcare staff with novel strategies ${ }^{6}$. Additionally, screening has expanded to beyond active case searches in younger ages and now evaluates the impact of RHD on pregnancy ${ }^{7}$ and investigates the susceptibility of siblings of positive cases $^{8}$, thereby unveiling previously unexplored territories. In addition, whilst most of the doubts surrounding echo screening are due to the uncertain prognostic meaning of "subclinical RHD" as defined by the 2012 World Heart Federation (WHF) criteria - as emphasized in the current review - efforts have been made to identify individuals at higher risk of disease progression. In Uganda, a subdivision of the "definite" group was proposed based on a robust follow-up program, and a predictive score was derived from a screening program in Brazil and validated in Ugandan cohorts with considerable accuracy ${ }^{1}$. In both cases, the importance of morphological abnormalities over functional findings have been demonstrated. However, the association of the proposed score with progression to clinical RHD and its external validation in other regions is still lacking and remain important limitations of the strategy. And, more importantly, the ongoing GOAL (Gwoko Adunu pa Lutino; clinicaltrials.gov No. NCT03346525) trial will add a crucial piece to the puzzle: the evaluation of the 
impact of secondary prophylaxis in patients with latent (definite) RHD and high-risk echo variables. Testing the hypothesis that penicillin will reduce the echo progression rate by $50 \%$ over 2 years, the study will go deeper into the relevance of screening as a public policy. Until that point, its broad implementation should not expand past the boundaries of research, especially if the potential stigmatization posed by a diagnosis of uncertain meaning is considered.

Broad preventive strategies are key to reducing RHD burden in the long run. Approaches at intervention are complex and multifactorial since RHD is closely associated with social issues such as poverty, overcrowding and poor access to healthcare, as demonstrated by the Global Burden of Disease ${ }^{1}$, with disproportionate trends in lower income regions. It must also be noted that some successful strategies have been reported with the integration of RHD control and existing health systems. Cuba has observed a dramatic drop in premature morbidity and mortality in children and young adults with a cost-saving intervention, with synergism between primary and secondary care $^{9}$. In Brazil, the integration of education and screening into primary care seems to achieve better coverage and engagement, with an important role played by community health agents ${ }^{6}$. Similarly, in Uganda, the regionalization and decentralization of RHD care to the community level is proposed as a tool for improving retention in care ${ }^{7,8}$. Additionally, research also focused on strategies to decentralize diagnostic services through pointof-care technologies (e.g. portable/handheld echocardiography and rapid antigen detection test kits), task-shifting and telemedicine, which may optimize case detection in areas with limited technology and skilled personnel and favor further integration with healthcare systems ${ }^{6}$. These investigations are of fundamental importance for future planning of healthcare actions and for reassuring the need for developing diagonal approaches.

On the other side of the disease spectrum, it must also be acknowledged that international RHD collaborations have resulted in better conditions for treatment of late sequelae and advanced valve/heart disease in several ways, most distinctly in the poorest regions. For example, inter-institutional partnerships and international grants have propitiated building surgical and interventional infrastructure in Africa ${ }^{7,8}$, and international training of cardiovascular teams in Brazil and Africa has helped the development of important percutaneous valve interventions programs. Furthermore, partnerships with medical device manufacturers have widened the availability of valve prostheses and interventional devices in areas where public funding is scarce. In parallel, these collaborations created conditions for further research initiatives with focus on severe valve disease. As it may take decades for preventive programs and public policies to bring about a reduction of advanced RHD rates, improving conditions to manage late sequelae may result in better outcomes and lower mortality in the mid-term.

Finally, the combination of the growing research interest and advocacy (and their possible synergic effect on societal and political awareness) gradually gave rise to significant advances towards high-level policy. Prior to the recent discussions in the WHO - as mentioned by Leal and cols - calls-to-action, including
4 fundamental statements (all from Africa) were published in about a decade, with great contributions to spread concepts related to RHD control. However, the ultimate goal was achieved in 2018 when the World Health Assembly adopted a resolution co-sponsored by countries from all $6 \mathrm{WHO}$ regions marking ARF and RHD global health priorities worldwide for the first time. Of outstanding importance for policymaking regarding RHD prevention and management, the resolution was celebrated as a demonstration of the urgent need for a global response.

There are some examples of progresses already been achieved towards the WHO resolution recommendations. As mentioned, incorporation of RHD into primary care priorities is being implemented in some African countries, as well as improved access to basic healthcare. There is growing engagement on international collaborations, involving joint actions, advocacy, registries and education, such as the RHD Action, the WHF RHD roadmap - a general guide and framework tool designed to help improve prevention and control of RHD - and RhEACH, a technical support and policy translation initiative to amplify broad RHD control efforts ${ }^{10}$. Besides educational tools for communities and healthcare workers, these collaborations involve comprehensive registerbased programs with great potential to reduce RHD burden at a reasonable cost in disparate geographic settings, also boosting research interest and, consequently, funding. Moreover, results of global registries such as the REMEDY ${ }^{10}$ have already been used to guide healthcare and research priorities, and highlight the urgent need for action on RHD social determinants, viz. poverty, education, access to health, basic sanitation, and living conditions. After all, it must be acknowledged that, besides being a health condition, RHD is a deep social issue on the world stage and must be faced as such.

\section{Conflict of Interests}

The authors have no competing interests to declare.

The corresponding author has the right to grant, on behalf of all authors, and does grant on behalf of all authors, an exclusive license (or non-exclusive for government employees) on a worldwide basis to the current publishing group and its licensees to permit this article (if accepted) to be published in the Journal of the Brazilian Society of Tropical Medicine editions and any other products to exploit all subsidiary rights.

\section{REFERENCES}

1. Nunes MCP, Sable C, Nascimento BR, Lima EM, da Silva JLP, Diamantino AC, Oliveira KKB, Okello E, Aliku T, Lwabi P, Colosimo EA, Ribeiro ALP and Beaton AZ. Simplified Echocardiography Screening Criteria for Diagnosing and Predicting Progression of Latent Rheumatic Heart Disease. Circ Cardiovasc Imaging. 2019;12:e007928.

2. Gray LA, D'Antoine HA, Tong SYC, McKinnon M, Bessarab D, Brown N, et al. Genome-Wide Analysis of Genetic Risk Factors for Rheumatic Heart Disease in Aboriginal Australians Provides Support for Pathogenic Molecular Mimicry. J Infect Dis. 2017;216(11):1460-70. 
3. Parks T, Mirabel MM, Kado J, Auckland K, Nowak J, Rautanen A, et al. Association between a common immunoglobulin heavy chain allele and rheumatic heart disease risk in Oceania. Nat Commun. 2017;8:14946

4. Shulman ST, Tanz RR, Dale JB, Steer AC and Smeesters PR. Added value of the emm-cluster typing system to analyze group A Streptococcus epidemiology in high-income settings. Clin Infect Dis. 2014;59(11):1651-2.

5. Tartof SY, Reis JN, Andrade AN, Ramos RT, Reis MG and Riley LW. Factors associated with Group A Streptococcus emm type diversification in a large urban setting in Brazil: a cross-sectional study. BMC Infect Dis. 2010;10:327.

6. Nascimento BR, Sable C, Nunes MCP, Diamantino AC, Oliveira $\mathrm{KKB}$, Oliveira CM, et al. Comparison Between Different Strategies of Rheumatic Heart Disease Echocardiographic Screening in Brazil: Data From the PROVAR (Rheumatic Valve Disease Screening Program) Study. J Am Heart Assoc. 2018;7(4).
7. Beaton A, Okello E, Scheel A, DeWyer A, Ssembatya R, Baaka O, et al. Impact of heart disease on maternal, fetal and neonatal outcomes in a low-resource setting. Heart. 2018;105(10):755-60.

8. Aliku T, Sable C, Scheel A, Tompsett A, Lwabi P, Okello E, et al. Targeted Echocardiographic Screening for Latent Rheumatic Heart Disease in Northern Uganda: Evaluating Familial Risk Following Identification of an Index Case. PLoS Negl Trop Dis. 2016;10(6):e0004727.

9. Watkins DA, Mvundura M, Nordet P and Mayosi BM. A costeffectiveness analysis of a program to control rheumatic fever and rheumatic heart disease in Pinar del Rio, Cuba. PloS one. 2015;10(3):e0121363.

10. Salinas A, Gonzalez G and Manuel Ramos J. Rheumatic Fever and Rheumatic Heart Disease: Collaboration Patterns and Research Core Topics. The Journal of heart valve disease. 2016;25(5): 619-27. 